\title{
Co-Mg Alaşımlarının Borlama Yöntemi ile Kaplanması ve Mikroyapısal ve Mekanik Özelliklerinin İncelenmesi
}

\author{
İsmail Yıldız ${ }^{1 *}$, İbrahim Güneş² \\ 1* Afyon Kocatepe Üniversitesi, İscehisar Meslek Yüksekokulu, Makine ve Metal Teknolojileri Bölümü, 03750, \\ Afyonkarahisar/Türkiye (ORCID: 0000-0002-9207-591X), iyildiz@aku.edu.tr \\ ${ }^{2}$ Giresun Üniversitesi, Mühendislik Fakültesi, İnşaat Mühendisliği Bölümü, 28200, Giresun/Türkiye (ORCID \\ 0000-0001-7595-0121), ibrahim.gunes@giresun.edu.tr
}

(İlk Geliş Tarihi 23.12.2021 ve Kabul Tarihi 30.12.2021)

(DOI: 10.29228/JCHAR. )

ATIF: Yıldız, I., Güneş, I., Borlanmış Co-Mg Alaşımlarının Yüzey Özelliklerinin İncelenmesi. Journal of Characterization, (1), Özel Sayı, pp 1-7, 2021.

$\ddot{O} \mathbf{z}$

Yapılan çalışmada, Co-Mg alaşımına (\%95 Co ve $\% 5 \mathrm{Mg}$ kompozisyon) borlama yöntemi ile kaplama yapılmıştır. Co ve Mg metal tozları faz diyagramındaki yüzdelik dilimlere göre kompozisyon karışım oranları belirlenmiştir. Belirlenen oranlarla kapalı kaplar içerisine konulan toz karışımlar 24 saat süre ile döner karıştırıcıda karışmıştır. Homojen karışım daha sonra geometrik şekle sahip olması için kalıplara dökülerek preslenmiştir. Preslenmiş numuneler kontrollü atmosferik firın içerisine konularak $530{ }^{\circ} \mathrm{C}$ sıcaklıkta sinterlenmiş̧ir. $\mathrm{Bu}$ işlem sonrasında numuneler borlama işlemi yapılması için silindirik olarak tasarlanmış kutular içerisinde alt ve üst taraflarına Ekabor II tozu dökülüp hava almaması için şamot çamuru ile kapatılarak $900{ }^{\circ} \mathrm{C}$ sıcaklık ve $1.5-4.5$ saat değişen sürelerde borlama işlemi yapılmıştır. Borlanmış numunelere en son XRD ve SEM analizlerin yanı sıra mikrosertlik testleri yapılmışır. XRD analiz sonucunda $\mathrm{CoB}, \mathrm{Co}_{2} \mathrm{~B}$ ve $\mathrm{Co}$ fazları ortaya çıkmıştır. SEM analiz sonrasında ise bor tabakaları oluşmuş ve tabaka kalınlık ölçümleri sonucunda en yüksek değer 4.5 saat süre sonrasında $120 \mu \mathrm{m}$ olarak bulunmuştur. Mikrosertlik ölçümü sonrasında ise en yüksek değer $1930 \mathrm{HV}_{0,05}$ olarak ölçülmüştür.

Anahtar Kelimeler: Co-Mg alaşım, borlama, mikrosertlik, XRD, SEM

\section{Coating of Co-Mg Alloys by Boriding Method and Investigation of Microstructural and Mechanical Properties}

\begin{abstract}
In the study, the Co-Mg alloy (95\% Co and 5\% $\mathrm{Mg}$ composition) was coated by boriding method. Composition mixing ratios were determined according to percentiles in the phase diagram of Co and $\mathrm{Mg}$ metal powders. Powder mixtures placed in closed containers at the determined rates were mixed in a rotary mixer for 24 hours. The homogeneous mixture was then poured into molds and pressed to form a geometric shape. Pressed samples were placed in a controlled atmospheric furnace and sintered at $530{ }^{\circ} \mathrm{C}$. After this process, the pieces were borided at a temperature of $900{ }^{\circ} \mathrm{C}$ and for $1.5-4.5$ hours by pouring Ekabor II powder on the top and bottom of the cylindrical designed boxes for boriding, and covered with chamotte mud to prevent air leakage. Performed the latest XRD and SEM analyses as well as microhardness tests
\end{abstract}


were performed on the borided samples. As a result of XRD analysis, $\mathrm{CoB}, \mathrm{Co}_{2} \mathrm{~B}$, and $\mathrm{Co}$ phases emerged. After SEM analysis, boron layers formed, and the highest value was found as $120 \mu \mathrm{m}$ after 4.5 hours due to layer thickness measurements. After the microhardness measurement, the highest value measured was $1930 \mathrm{HV}_{0.05}$.

Keywords: Co-Mg alloy, boriding, microhardness, XRD, SEM

\section{Giriş}

Malzemelerin yüzey direncini artırma yöntemlerden olan elektrokimyasal yöntemler arasında borlama yer almaktadır [1-5]. Aşınmanın yanı sıra korozyon, yorulma ve oksidasyon direnci gibi önemli özelliklere sahip olması nedeniyle diğer yöntemlere göre avantajlar vardır [6,7]. Borlama yönteminin uygun sıcaklıklar ve sürelerde yapılmasıyla kaplanan malzemelerin mekanik özellikleri iyi hale gelmektedir. Kaplama ortamında oksitleyici durumların olmaması için çok iyi vakum yapılması gerekmektedir [8].

Co bazlı alaşımlar kullanıldıkları ortamlarda önemli özelliklere sahip olmaları nedeniyle en çok tercih edilen alaşımları arasındadır [9,10]. Bu özellikler arasında, yüksek yorulma dayanımı, aşınma ve korozyon direnci gibi özellikler gelmektedir. $\mathrm{Bu}$ alaşımlara uygulanan farklı kaplama yöntemleri ile mevcut özelliklerinde daha da iyileşmeler olmaktadır [11]. Kaplama yöntemleri arasında yer alan borlama, bor atomlarının ana malzeme olarak metal ya da alaşım yüzeyine difüzyon etmesi yoluyla yüzeylerde borür tabakasının oluşması sonrasında elde edilmektedir. Burada önemli olan uygulanan borlama sıcaklığı, malzemelerin kimyasal bileşimi, bor materyali ve borlama sonrasında $\mathrm{FeB}, \mathrm{Fe}_{2} \mathrm{~B}$ ve $\mathrm{CoB}, \mathrm{Co} 2 \mathrm{~B}$ gibi metalik fazlar oluşmaktadır $[12,13]$.

Gerçekleşen çalışmada sonucunda, Co-Mg alaşımlarına borlama yönteminin etkileri incelenmiştir. Sinterleme sonrasında elde edilen numunelere kutu borlama yöntemi ile borlama işlemi yapılmıştır. Bu işlem sonucunda oluşan bor tabakaları SEM ve XRD analiz ile belirlenmiş, vickers sertlik testleri yapılarak sertlikleri ölçülmüş ve yüzeylerde oluşan yapılar tespit edilmiştir.

\section{Materyal ve Metot}

Yapılan çalışmada, \% 99,5 saflığa sahip Co ve Mg metal tozları ile Co-Mg alaşımları (\%95 Co-\%5 Mg kompozisyon) oluşturulmuştur. Mikron boyutundaki bu tozlar homojen bir şekilde karıştırılmaları için döner bir karıştırıcıda 24 saat süre ile kapalı kutu içerisinde karıştırılmıştır. Karışan tozlar dairesel şekle sahip olmak için özel olarak hazırlanmış metal kalıplara dökülerek şekillendirilmiştir. Bu işlem yaklaşık olarak $25 \mathrm{~N}$ kuvvet sonucunda yapılmıştır. Ortaya çıkan numunelere $530{ }^{\circ} \mathrm{C}$ sıcaklıkta 2 saat süreyle 1 sil işlem uygulanmıştır.

Isıl işlem sonrasında dairesel numuneler kapalı kutu içerisinde borlama yöntemi ile borlanmıştır. Bu yöntem, kontrollü atmosferik firın ortamında kapalı kutular içerisine konulan numunelerin alt ve üst kısımlarına Ekabor II toz dökülerek yapılmıştır (Şekil 1). Bu işlem için literatürde $800-1100{ }^{\circ} \mathrm{C}$ arası sıcaklıklarda borlama çalışmaları yapılmış [14,15], bu çalışmada ise $900{ }^{\circ} \mathrm{C}$ sıcaklık ve1,5-4,5 saat süre aralıklarda ısıl işlem uygulanmıştır. Isıl işlem sonrası fırından çıkarılan numuneler hava ile soğumasından sonra metalografik analiz olarak XRD ve SEM, vickers mikrosertlik testi yapılmıştır.

XRD analiz çalışmasında, alfa ışını, 0,02 \% dk tarama hızı ve 2 Theta tarama açısı uygulanmıştır. Numunelerin yoğunlukları $\mathrm{d}=\mathrm{m} / \mathrm{v}$ formülüne göre hesaplanmıştır. Burada $\mathrm{m}$, numune kütlesi; $\mathrm{v}$, numune hacmidir. Borlama sonucu yüzeylerde oluşan borür tabakarın sertlik ölçümleri SHIMADZU HMV-2 model sertlik cihazı ile yüzeyden merkeze doğru 50 gr. yük altında vickers yöntemiyle yüzeyden içeriye doğru 5 farklı ölçüm yapılarak ortalama sonuç elde edilmiştir. 


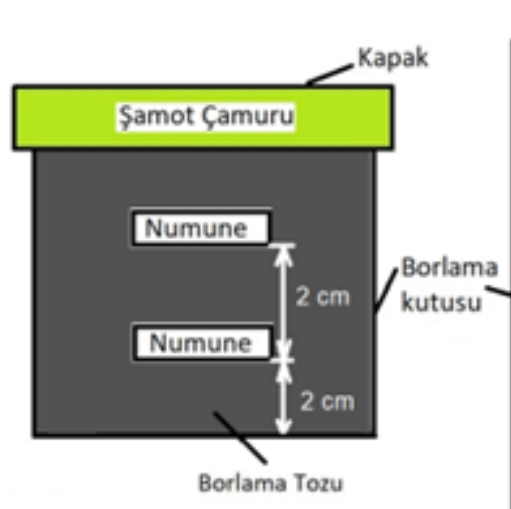

(a)

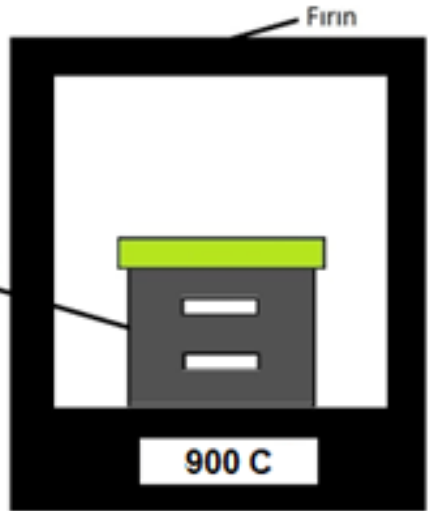

(b)

Şekil 1. Kapalı firın ortamında borlama işleminin yapılışı (a: Numunelerin kutu içerisine yerleştirilmesi, $b$ : Numunenin borlanmasi).

\section{Bulgular}

Co-Mg tozlarına sirasıyla presleme, sinterleme, yoğunluk, borlama ve metalografik analiz ile sertlik ölçümleri yapılmıştır. $530^{\circ} \mathrm{C}$ sıcaklıkta 2 saat süre ile yapılan sinterleme sonrasında yoğunluk ölçümü ile Co-Mg alaşımında yoğunluk $7,21 \mathrm{gr} / \mathrm{cm}^{3}$ olarak ortaya çıkmıştır. Borlama işlemi $900{ }^{\circ} \mathrm{C}$ sıcaklık ve $1.5-$ 4.5 saat sürelerde yapılmıştır (Şekil 2).

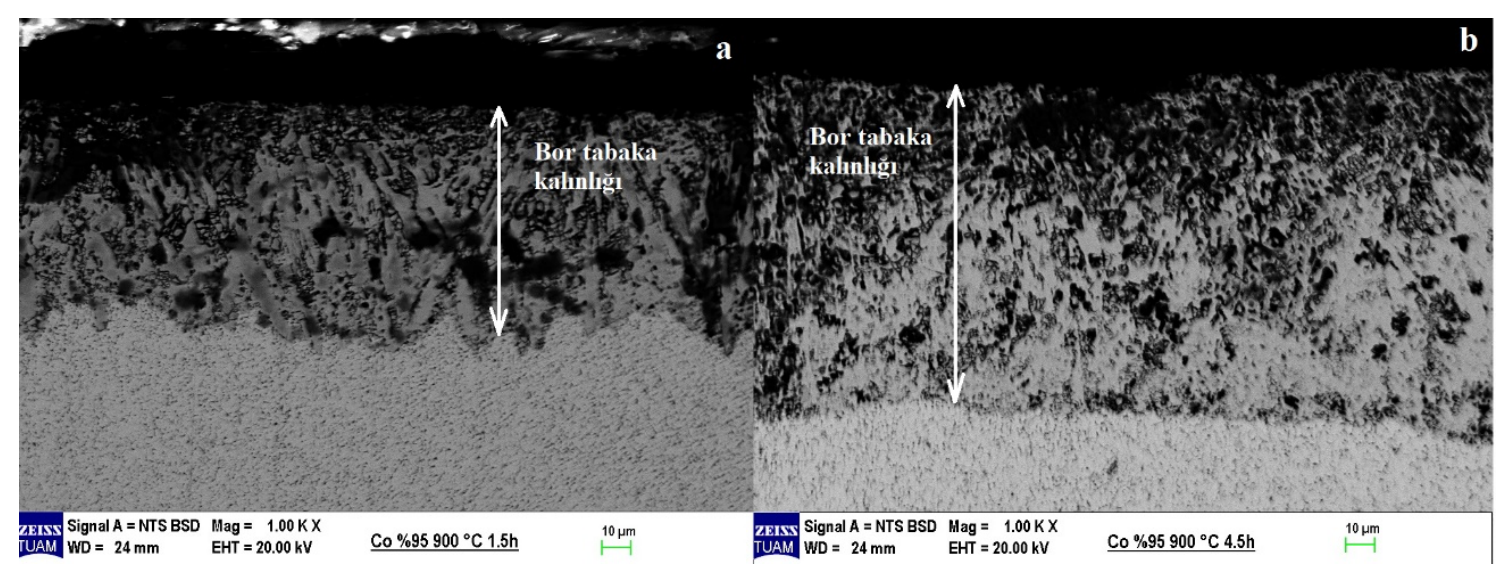

Şekil 2. Borlanmış \% 95 Co ile \% 5 Mg alaşımının mikroyapı görüntüleri (a: $900{ }^{\circ} \mathrm{C} 1,5$ saat, b: $900{ }^{\circ} \mathrm{C}$ 4,5 saat)

Borlama sonrasında numunelere metalografik analiz olarak XRD ve SEM analiz yapılmıștır. XRD analiz sonucunda $\mathrm{CoB}, \mathrm{Co}_{2} \mathrm{~B}$ ve $\mathrm{Co}$ faz yapıları ortaya çıkmıştır (Şekil 3). Sıcaklık ve süre artışına bağlı olarak $\mathrm{CoB}, \mathrm{Co}_{2} \mathrm{~B}$ faz değerleri 2000 ve 2500 değerlerinde bulunmuştur. Johnston vd., Campos-Silva vd. yaptıkları borlama çalışmalarını $[16,17], 950$ ve $1000^{\circ} \mathrm{C}$ 'de gerçekleştirmiş, oluşan bor tabakaları için XRD sonucunda $\mathrm{CoB}$ ve $\mathrm{Co}_{2} \mathrm{~B}$ faz yapılarını 1500 yoğunluk değerine yakın olarak elde etmişlerdir. SEM analiz çalışması sonrası en iyi mikroyapı ve bor katmanı Şekil 2(b)'de de görüleceği üzere $900{ }^{\circ} \mathrm{C}$ sıcaklık 4.5 saat süre sonrasında ortaya çıkmış ve yapılan bor tabaka kalınlığı ölçüm sonucunda $120 \mu \mathrm{m}$ kalınlık ölçülmüsşür. Mikrosertlik ölçümü için numune üzerinden kaplama bölgelerinden 5 farklı ölçüm yapılarak $1930 \mathrm{HV}_{0,05}$ değer ölçülmüştür (Şekil 4). 
Journal of Characterization
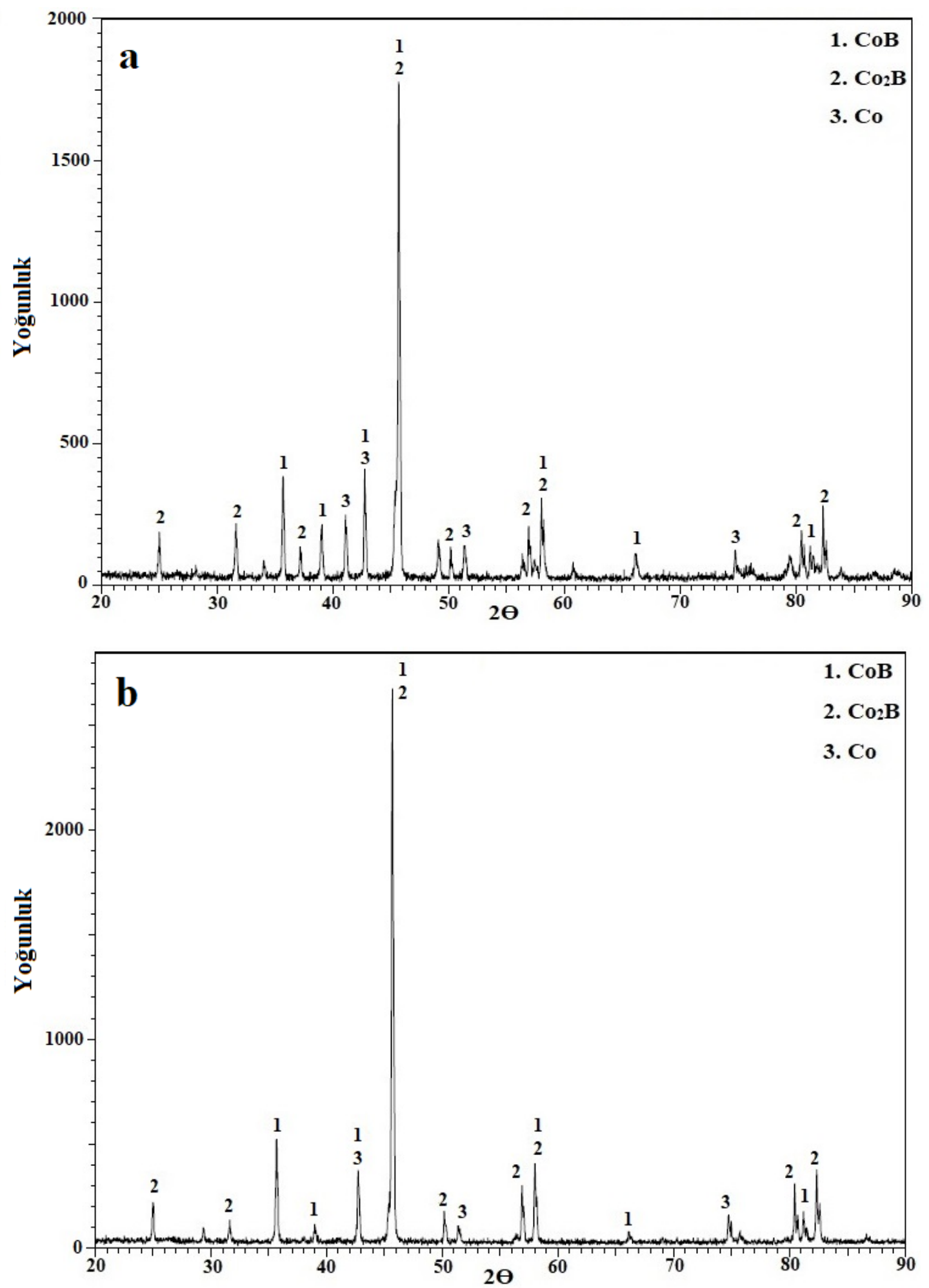

Şekil 3. Borlanmış \% 95 Co ile \% $5 \mathrm{Mg}$ alaşımının XRD analiz görüntüleri (a: $900{ }^{\circ} \mathrm{C} 1,5 \mathrm{saat}, b$ : $900{ }^{\circ} \mathrm{C}$ 4,5 saat) 


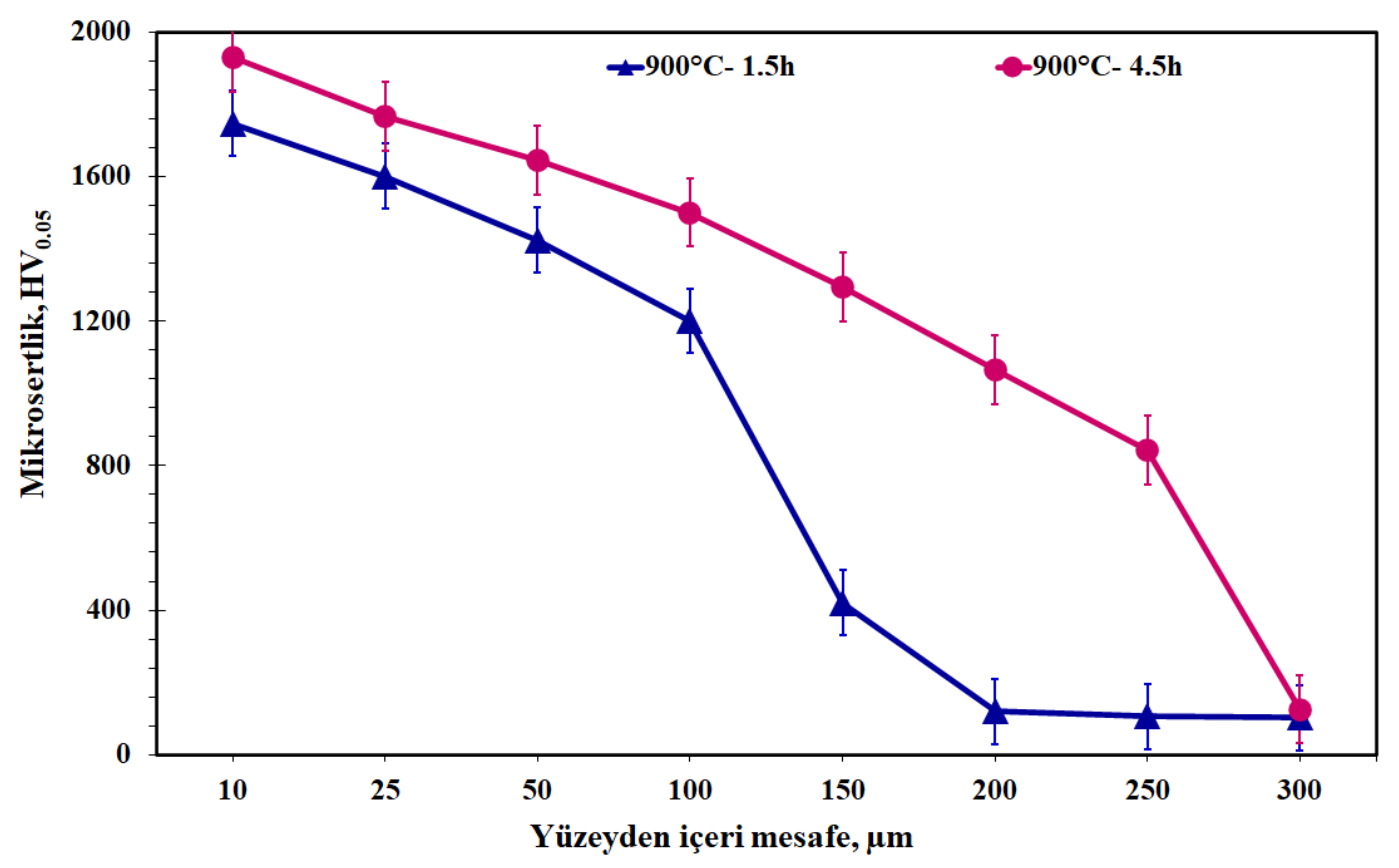

Şekil 4. Borlanmış \% 95 Co ile \% 5 Mg alaşımına yapılan mikrosertlik analiz sonucu

\section{Sonuçlar ve Tartışma}

Co-Mg alaşımının borlama sonrasında yapılan test ve analizler sonrasında ortaya çıkan sonuçlar aşağıda verilmiştir:

Borlama işlemi sonrasında en yüksek bor tabaka kalınlığ 4,5 saat süre sonrasında $120 \mu$ olarak bulunmuştur.

Co-Mg alaşımların sinterleme sonrası ölçülen yoğunluk değeri 7,21 gr/ $\mathrm{cm}^{3}$ olarak bulunmuştur.

Sinterleme sonrası yapılan sertlik testi sonucu $135 \pm 9 \mathrm{HV}_{0,05}$ değeri ortaya çıkmıştır.

Metalografik analiz olarak XRD analizi yapılmıştır. Analiz sonucu olarak $\mathrm{CoB}, \mathrm{Co}_{2} \mathrm{~B}$ ve $\mathrm{Co}$ faz yapıları elde edilmiştir. Bu yapıların değerleri sıcaklık ve süre artışına bağlı olarak artmıştır. Bu durum borlamanın olduğunu göstermiştir.

Borlama sonrası yapılan mikrosertlik testi sonucu numunelerde sırasıyla $1820 \mathrm{HV}_{0,05}$ ve $1930 \mathrm{HV}_{0,05}$ sertlik değerleri ölçülmüştür.

Gerçekleşen borlama yönteminde sıcaklık ve süreye paralel olarak borür tabakaları belirgin hale gelmiş, tabaka kalınlarında artışlar olmuştur. $900{ }^{\circ} \mathrm{C}$, ortaya çıkan sonuçlara göre en iyi borlama sıcaklığı olarak belirlemiştir.

Alaşım içerisinde bulunan $\mathrm{Mg}$, hava ile temasa geçtiğinde çok çabuk bozulabilen bir malzemedir. Yapılan sinterleme ve borlama çalışmalarında yapıda bozulmaların oluşması $\mathrm{Mg}$ malzemesinden kaynaklanmıştır. Kapalı vakum ortamına sahip sistem içerisinde hem sinterleme hem de borlamanın yapılması daha uygun olacaktır.

\section{Teșekkür}

Yapılan çalışma, Afyon Kocatepe Üniversitesi Bilimsel Araştırma Projeleri’nden 17.MYO.05 no'lu Genel Amaçlı proje ile desteklenmiştir. Desteklerinden dolayı çok teşekkür ederim. 


\section{Kaynaklar}

[1] F. Hongze, L. Zhou, S. Wang, et al. "Formation and kinds of boride on microstructure evolution and mechanical properties in Ti47.5Al2.0Cr2.0NbxB alloys", Intermetallics, vol. 141, 107427, 2022.

[2] I. Yıldız, I. Gunes, "Borlanmış \% $5 \mathrm{Mg}$ katkılı Ni-Mg alaşımının yüzey özelliklerinin incelenmesi”, Politeknik Dergisi, vol. 23(1), pp. 97-104, 2020.

[3] C. T. Sezgin, F. Hayat, "The effects of boriding process on tribological properties and corrosive behavior of a novel high manganese steel", Journal of Materials Processing Technology, vol. 300, 117421, 2022.

[4] I. Yıldız, I. Gunes, "\%3,0 Mg içeren Borlanmış Co-Mg Alaşımının Yüzey Özelliklerinin İncelenmesi”, El-Cezerî Journal of Science and Engineering, vol: 6 (3), 533-542, 2019.

[5] M.A. Doñu-Ruiz, N. López-Perrusquia, A. Renteria-Salcedo, et al., "Tribocorrosion behavior of boride coating on CoCrMo alloy produced by thermochemical process in 0.35\% NaCl solution", Surface and Coatings Technology, vol. 425,127698, 2021.

[6] D. Fernández-Valdés, O. Vasquez-De la Rosa, G.A. Rodríguez-Castro, A. et al., "A numerical-experimental study of AISI 316L borided steels under cyclic contact loading", Surface and Coatings Technology, vol. 423, 127556, 2021.

[7] I. Gunes, I. Y1ldız, "Investigation of Adhesion and Tribological Behavior of Borided AISI 310 Stainless Steel”, Revista Materia, vol. 21, pp. 61-71, 2016.

[8] I. Gunes, A. G. Celik, "Surface Characterization of Borided S220 Rebar", Journal of Characterization, vol. 1(2), 66-70, 2021.

[9] X. Yang, C Li, Zhenhua Ye, X Zhang, et al., "Effects of tribo-film on wear resistance of additive manufactured cobalt-based alloys during the sliding process", Surface and Coatings Technology, vol. 427, 127784, 2021.

[10] H. Arabnejad, H. Uddin, K. Panda, et al., "Testing and modeling of particle size effect on erosion of steel and cobalt-based alloys", Powder Technology, vol. 394, 11861194, 2021.

[11] R. Kamalakannan, K. DineshKumar and K. NarenRaj, "The sliding wear behavior of $\mathrm{CrCuFeNi}$ alloyed with various combinations of cobalt", Materials Today: Proceedings, In Press, 2021.

[12] I. Gunes, I. Y1ld1z, "Rate of Growth of Boride Layers on Stainless Steels", Oxidation Communication, vol. 38(4/A), 2189-2198, 2015. 
[13] Li Li, Peng Liu, Yongzhi Zhou, Huamei Duan, Mujun Long, Dengfu Chen, Mechanical properties and electronic structures of borides in Ni-Co-B alloy system, Materials Letters, vol. 309,131312, 2022.

[14] C. T. Sezgin, F. Hayat, "The effects of boriding process on tribological properties and corrosive behavior of a novel high manganese steel", Journal of Materials Processing Technology, vol. 300, 117421, 2022.

[15] I. Campos-Silva, E. J. Hernández-Ramirez, A. Contreras-Hernández, et al. "PulsedDC powder-pack boriding: Growth kinetics of boride layers on an AISI 316 L stainless steel and Inconel 718 superalloy", Surface and Coatings Technology, vol. 421, 127404, 2021.

[16] J. M. Johnston, M. Jubinsky, S. A. Catledge, "Plasma boriding of a cobalt-chromium alloy as an interlayer for nanostructured diamond growth", Applied Surface Science, vol. 328, 133-139, 2015.

[17] I. Campos-Silva, D., Bravo-Bárcenas, H., Cimenoglu, et al., "The boriding process in CoCrMo alloy: Fracture toughness in cobalt boride coatings", Surface and Coating Technology, vol. 260, 362-368, 2014. 